\title{
LAS REFORMAS A LA LEY DE EXPROPIACIÓN RELACIONADAS CON LA GARANTÍA DE AUDIENCIA PREVIA
}

Marat PAREDES MONTIEL José María SOBERANES DíEZ

\section{INTRODUCCIÓN}

El presente trabajo tiene por objeto comentar las recientes reformas a la Ley de Expropiación ${ }^{1}$ a partir de la jurisprudencia de la Suprema Corte de Justicia de la Nación que interpretó este cuerpo legal a la luz de la garantía de audiencia.

La Ley de Expropiación proviene de 1936, desde entonces ha sufrido muy pocas modificaciones. En diciembre de 1949 se adicionó para que mencionara que la construcción de oficinas para el gobierno federal era causa de utilidad pública. En diciembre de 1993, con el NAFTA a entrar en vigor, se hicieron algunas modificaciones: a) se precisó un plazo de 45 días para que la autoridad resolviera sobre la reversión del bien; b) se le denominó decreto al ordenamiento por el que se dispone la expropiación, y c) también se indicó que el precio que se fijara como indemnización debería ser equivalente a su valor comercial, que debía pagarse en moneda y no en especie, reduciendo el plazo para el pago de diez a un año. En diciembre de 1997, en el contexto de los cambios legislativos que precedieron a la reforma constitucional sobre el Distrito Federal, se dispuso la facultad del jefe de gobierno del Distrito Federal de declarar la expropiación.

1 La reforma en comentario fue publicada en el Diario Oficial de la Federación del 5 de junio de 2009 . 
Después de estas reformas se encuentra la que es objeto del presente comentario, la cual tuvo por finalidad, entre otras, adecuar la ley, no a un contexto político-constitucional o a un entorno económico, sino a una interpretación realizada por el Pleno y la Segunda Sala de la Suprema Corte de Justicia de la Nación. Se trata de la obligación jurisprudencial de que rija la audiencia previa en los casos de expropiación.

Así pues, se analizará el contexto interpretativo en el que se desarrolló la reforma para después abordar el contenido de la misma.

\section{LA JURISPRUDENCIA SOBRE LA AUDIENCIA \\ EN LA EXPROPIACIÓN}

Apenas dos años después de la expedición en vigor de la Ley de Expropiación se cuestionó su constitucionalidad por no prever un procedimiento en el que se escuchara a los propietarios antes de ser privados de sus bienes. Se trató de los amparos promovidos por diversas compañías petroleras con motivo de la expropiación petrolera decretada por el presidente Cárdenas en $1938 .^{2}$

Del recurso de revisión de dichos amparos correspondió conocer a la Segunda Sala, ${ }^{3}$ cuando la misma conocía todavía de amparos contra leyes, previo a la reforma que trasladó dicha competencia en forma exclusiva al Tribunal Pleno. La Sala determinó que el artículo 27 constitucional, al referirse a la expropiación, no contemplaba la audiencia previa dentro las condiciones necesarias para su ejercicio. Por tanto, sostuvo no podía considerarse que dicha garantía rigiera en los casos de expropiación. ${ }^{4}$

Este criterio utiliza un método interpretativo recurrente en la jurisprudencia constitucional mexicana: si el Constituyente lo hubiese querido, así lo hubiera dicho. La interpretación del silencio del legislador es una de las formas que se ha encontrado para sostener decisiones sin mayor

2 Posiblemente el más famoso de estos asuntos haya sido el promovido por la Compañía Mexicana de Petróleo "El Aguila", S. A.

3 Los amparos en revisión 2902/39, 5446/39, 4652/39, 8054/39 y 4406/42 se fallaron, respectivamente, el 2 de diciembre de 1939, 28 de marzo de 1940, 29 de junio de 1940, 24 de septiembre de 1940 y 9 de octubre de 1942. En contra del criterio mayoritario votó el ministro José María Truchuelo, constituyente de 1916.

4 Tesis 834, Apéndice al Semanario Judicial de la Federación de 1917-1988, parte II, p. 1389. 
justificación. Es un criterio que sirve para ambas posiciones. Si el Constituyente hubiera querido la audiencia, la hubiera establecido; pero si hubiese querido que no rigiera, también lo hubiera dicho. Su enorme ductilidad permite al Tribunal relajar su obligación de motivación, disfrazando en ocasiones una decisión voluntarista.

Independientemente de la deficiencia interpretativa, la decisión de la Segunda Sala fue que la audiencia previa no regía en caso de expropiación. Ese fue un criterio que se reiteró en durante muchos años. Además del criterio publicado en la quinta época del Semanario Judicial de la Federación, el Pleno de la Suprema Corte dijo lo mismo en las siguientes oportunidades que tuvo de pronunciarse. ${ }^{5}$

Después de la reforma constitucional relativa al Poder Judicial de la Federación de diciembre de 1994, la Suprema Corte tuvo la oportunidad de pronunciarse respecto a este mismo tema. Al resolver unos amparos en revisión reiteró el criterio tradicional sustentado por el Tribunal Pleno, ${ }^{6}$ ordenando nuevamente la publicación de la jurisprudencia surgida de los asuntos antes mencionados. ${ }^{7}$

En dicho asunto se dieron sustancialmente tres razones para sostener que la garantía de audiencia previa no operaba en el caso de la expropiación:

1. La garantía de previa audiencia no es un requisito de los que se establecen en el artículo 27 constitucional para emitir actos expropiatorios.

2. El artículo 14 de la Constitución federal contiene una regla general para derechos individuales, mientras que el numeral 27 del mismo ordenamiento establece garantías sociales que, por su propia naturaleza, están por encima de aquéllos.

3. La expropiación obedece a circunstancias apremiantes que requieren una determinación rápida, la cual no podría lograrse oyendo al afectado previamente a su urgente ejecución.

5 Por ejemplo, en la tesis de rubro "EXPROPIACION, LA GARANTÍA DE AUDIENCIA NO RIGE EN MATERIA DE”, formada con motivo de amparos resueltos entre 1962 y 1973, publicado once ocasiones distintas, la última en el Apéndice al Semanario Judicial de la Federación 1917-1995, t. III, materia administrativa, p. 46.

6 Amparos en revisión 54/91 y 446/96, resueltos en la sesión del 27 de febrero de 1997.

Cfr. Semanario Judicial de la Federación y su Gaceta, novena época, t. V, junio de 1997, p. 44. A la nueva publicación se le dio el número de jurisprudencia P./J. 65/95. 
En aquella ocasión, a pesar de que se reiteró en lo fundamental el contenido de la jurisprudencia del Pleno, se presentó una importante disidencia formada por los ministros Aguirre Anguiano, Góngora Pimentel, Gudiño Pelayo y Silva Meza.

El criterio sobre la operación de la audiencia en la expropiación volvió a ser revisado en enero de 2006, al conocer los amparos en revisión 1133/2004, 1132/2004, 1131/2004. En ellos un grupo de cañeros impugnaba la constitucionalidad de la Ley de Expropiación por las mismas razones que en todos los casos anteriores. Con base en esos asuntos, el Pleno de la Suprema Corte de Justicia de la Nación cambió de criterio, interrumpiendo su jurisprudencia anterior, que había resistido varios lustros.

Los motivos para el cambio de criterio son, en síntesis, los siguientes:

1. La potestad expropiatoria no implica que la Constitución autorice actuaciones arbitrarias de los poderes públicos que dejen sin efectos las garantías. Del artículo 27 constitucional no se desprende que la Constitución haya establecido una excepción expresa de la garantía de audiencia previa tratándose de la expropiación, sino que solamente existe un silencio constitucional a ese respecto.

2. Por ello, el contenido del artículo 27 constitucional debe relacionarse con el segundo párrafo del artículo 14 de dicho ordenamiento, tomando en cuenta que tratándose de actos privativos, la defensa debe ser previa para que sea adecuada y efectiva.

3. Aunque haya materias en que exista una audiencia posterior al acto privativo, como en los impuestos, en el caso de la expropiación no se justifica ello porque la eficacia de la defensa se ve mermada por el transcurso del tiempo, llegando a hacer imposible la recuperación de los bienes concretos que han sido objeto de la privación.

Estos casos, a pesar de que contaban una mayoría de ocho votos suficientes para lograr la interrupción de la jurisprudencia ${ }^{8}$ y de diez en el sentido de que en el caso impugnado el precepto era inconstitucional, no

8 Ministros Aguirre Anguiano, Luna Ramos, Góngora Pimentel, Gudiño Pelayo, Ortiz Mayagoitia, Sánchez Cordero, Silva Meza y presidente Azuela Güitrón. El ministro Cossío Díaz estimó que la audiencia puede ser previa o posterior y en pro de la reiteración del antiguo criterio estuvieron los ministros Díaz Romero y Valls Hernández. 
fueron los cinco precedentes necesarios para formar una nueva jurisprudencia por reiteración en sentido contrario. Por ese motivo, el presidente de la Suprema Corte, a partir de esos casos concretos, solicitó a la Segunda Sala que modificara la jurisprudencia sustentada a partir de la expropiación petrolera. Como la Segunda Sala era integrada por cuatro de los seis ministros que votaron a favor de la audiencia previa en la expropiación, se dio el cambio de criterio. ${ }^{9}$ De esta forma, es jurisprudencia firme de la Segunda Sala que dicha garantía debe otorgarse de manera previa en los procedimientos de expropiación. ${ }^{10}$

Este cambio de criterio ha sido criticado por Carlos Elizondo Mayer-Serra y Luis Manuel Pérez de Acha. ${ }^{11}$ Estos autores exponen que si bien resulta razonable que la expropiación requiera audiencia previa evitar la arbitrariedad de la que fueron víctimas muchos individuos durante décadas, lo cierto es que puede haber sido la puntilla final a la capacidad del Estado de volver a hacer una obra importante que requiere terrenos en manos de privados que deben ser expropiados.

Señalan que se trató de una interpretación radicalmente garantista de la Constitución, que no cuidó las implicaciones de los amparos concedidos. En efecto, apuntan que la nueva interpretación no contempló que no existen procedimientos bien definidos para dar la audiencia previa por lo que se abre un nuevo mundo de recursos en manos del afectado para buscar frenar una expropiación.

La posición anterior se funda en una concepción utilitarista de los derechos que no compartimos. Unos derechos que se tienen sólo en la medida en que no se considere útil violarlos, únicamente pueden ser llamados derechos de manera impropia. ${ }^{12}$ Sin embargo, coincidimos con dichos autores en el disenso con la postura de la Suprema Corte.

9 Así lo resolvió el 18 de agosto de 2006, por mayoría de cuatro votos y con la disidencia del ministro Juan Díaz Romero.

10 De este asunto surgió la jurisprudencia 2a./J. 124/2006, de rubro "EXPROPIACIÓN. LA GARANTÍA DE AUDIENCIA DEBE RESPETARSE EN FORMA PREVIA A LA EMISIÓN DEL DECRETO RELATIVO", Semanario Judicial de la Federación y su Gaceta, t. XXIV, septiembre de 2006, p. 278.

11 Elizondo, Carlos y Pérez de Acha, Luis Manuel, ¿Un nuevo derecho o el debilitamiento del Estado? Garantía de audiencia previa en la expropiación, supra, pp. 99-147.

12 Cfr. Massini, Carlos I., Los derechos humanos en el pensamiento actual, Buenos Aires, Abeledo-Perrot, 1994, p. 60. 
En primer término, porque parte de la misma falacia interpretativa del criterio anterior. Al señalar que del artículo 27 constitucional no se desprende que la Constitución haya establecido una excepción expresa de la garantía de audiencia previa tratándose de la expropiación, se está recurriendo al argumento de que "si el Constituyente hubiera querido así lo hubiera dicho". El silencio del legislador no puede ser la premisa de ninguna postura.

En segundo término, porque a la audiencia se le da un carácter sustancial que no tiene. ${ }^{13}$ Los artículos 14 y 27 constitucionales reconocen el derecho a la propiedad privada. La audiencia previa no es más que un procedimiento, un requisito para su limitación por el poder público. La expropiación es otro de los procedimientos constitucionales con los que se puede limitar la propiedad. En ese sentido, la cuestión a debate no es si se puede expropiar menoscabando derechos fundamentales, sino si el procedimiento constitucional de expropiación incluye la formalidad a la que alude el 14 constitucional.

En tercer término, porque la respuesta al cuestionamiento anterior parte del presupuesto de que no podría repararse la violación constitucional. Esto es contradictorio con la misma sentencia que no dispone una "nulidad absoluta" de la expropiación, sino una "nulidad para efectos" en tanto permite la expropiación siempre y cuando se les dé audiencia a los quejosos conforme a la "tesis Fraga". ${ }^{14}$ Este problema, además, no tiene que ver con la interpretación de derechos. Es una cuestión referente a la medida cautelar conservativa que debe existir en el respectivo juicio de amparo.

Siendo la cuestión a debatir si el procedimiento constitucional de expropiación incluye la formalidad a la que alude el 14 constitucional, debemos apuntar que consideramos existe una delegación legislativa para responder esta problemática, como apuntó el ministro Cossío Díaz en su voto concurrente. El segundo párrafo de la fracción VI del artículo 27 constitucional establece que la Federación o los estados, en sus respecti-

13 Sobre la incorrecta interpretación del artículo 14 constitucional que encuentra en éste un derecho constitucional, Rabasa, Emilio, El artículo 14 y el juicio constitucional, México, Porrúa, 1993, p. 89; Fix-Zamudio, Héctor, Ensayos sobre el derecho de amparo, México, Porrúa, 2003, p. 622.

14 Se conoce así a la tesis de rubro "AUDIENCIA, GARANTÍA DE", visible en el Semanario Judicial de la Federación, quinta época, t. LXXX, p. 3819, en virtud de que el ministro ponente de la misma fue Gabino Fraga. 
vas competencias determinarán el procedimiento administrativo para efectuar la expropiación. ${ }^{15}$

En el procedimiento administrativo que debe idear el legislador deben cumplirse, desde luego, con las previsiones constitucionales. En este sentido, siempre se debe regular un procedimiento de defensa de los afectados por la expropiación. Se trata de una exigencia derivada del principio de Estado de derecho y del derecho a la tutela judicial efectiva. Sin embargo, la posibilidad de defensa puede ser anterior o posterior. En esto existe una libertad de configuración legislativa.

No obstante, como toda medida en la que el legislador cuente con libertad, sus decisiones no pueden ser arbitrarias. La determinación de cuándo no se debe escuchar previamente al propietario del bien afectado, debe ser justificada en tanto está limitada por el principio de razonabilidad que debe informar todas la actuación del legislador. En un desastre natural está justificado que no se escuche previamente a los afectados con la expropiación, porque se tiene que hacer frente a una emergencia. Pero ante una obra vial, que tarda meses en planearse, podría optar por escuchar previamente el parecer de los afectados.

\section{LAS REFORMAS DE JUNIO DE 2009}

El 5 de junio de 2009 fueron publicadas en el Diario Oficial de la Federación las reformas a la Ley de Expropiación, es decir, dos años y medio después de la determinación de la Suprema Corte de Justicia de la Nación.

No deja de parecer interesante que en la evaluación costo-beneficio tomada por el órgano legislativo, se consideró más benéfico realizar la reforma a la ley, regulando la audiencia previa, que continuar con una disposición declarada inconstitucional por un criterio mayoritario del Pleno y por la jurisprudencia de la Segunda Sala de la Suprema Corte de Justicia de la Nación, siendo una de las pocas ocasiones en que un golpe

15 Textualmente dispone "Las leyes de la Federación y de los estados en sus respectivas jurisdicciones, determinarán los casos en que sea de utilidad pública la ocupación de la propiedad privada, y de acuerdo con dichas leyes la autoridad administrativa hará la declaración correspondiente..."; al señalar que de acuerdo con las leyes se llevará a cabo el procedimiento administrativo, entendemos que se delega la configuración de la forma al legislador ordinario. 
de jurisprudencia conlleva la reforma de una ley, en un tiempo razonablemente corto.

¿Cuál es la fórmula adoptada en la reforma a la Ley de Expropiación?

En primer lugar, se decide que la audiencia previa debe ser realizada en sede administrativa y no ante autoridad judicial, lo cual nos parece importante para asegurar la agilidad del procedimiento.

Por otra parte, el procedimiento administrativo de expropiación se divide en dos etapas claramente diferenciadas:

a) La declaración de utilidad pública en donde se materializa la garantía de audiencia previa a los particulares.

b) La emisión del decreto expropiatorio en donde se fija, en principio, el monto de la indemnización.

El procedimiento de declaración de utilidad pública se desarrolla de la manera siguiente: ${ }^{16}$

16 Artículo 2o. En los casos comprendidos en el artículo anterior, la Secretaría de Estado competente emitirá la declaratoria de utilidad pública, conforme a lo siguiente:

I. La causa de utilidad pública se acreditará con base en los dictámenes técnicos correspondientes.

II. La declaratoria de utilidad pública se publicará en el Diario Oficial de la Federación y, en su caso, en un diario de la localidad de que se trate, y se notificará personalmente a los titulares de los bienes y derechos que resultarían afectados.

En caso de ignorarse quiénes son los titulares o bien su domicilio o localización, surtirá efectos de notificación personal una segunda publicación de la declaratoria en el Diario Oficial de la Federación, misma que deberá realizarse dentro de los cinco días hábiles siguientes a la primera publicación.

III. Los interesados tendrán un plazo de quince días hábiles a partir de la notificación o de la segunda publicación en el Diario Oficial de la Federación para manifestar ante la Secretaría de Estado correspondiente lo que a su derecho convenga y presentar las pruebas que estimen pertinentes.

IV. En su caso, la autoridad citará a una audiencia para el desahogo de pruebas, misma que deberá verificarse dentro de los ocho días hábiles siguientes a la recepción de las manifestaciones a que se refiere el párrafo anterior. Concluida dicha audiencia, se otorgará un plazo de tres días hábiles para presentar alegatos de manera escrita.

V. Presentados los alegatos o transcurrido el plazo para ello sin que se presentaren, la autoridad contará con un plazo de diez días hábiles para confirmar, modificar o revocar la declaratoria de utilidad pública.

La resolución a que se refiere la fracción anterior no admitirá recurso administrativo alguno y solamente podrá ser impugnada a través del juicio de amparo.

VII. El Ejecutivo federal deberá decretar la expropiación a que se refiere el artículo 4o. de esta Ley, dentro de los treinta días hábiles siguientes a que se haya dictado la resolución señalada en la fracción $\mathrm{V}$ que antecede. Transcurrido el plazo sin que se haya 
a) La Secretaría de Estado competente deberá integrar y tramitar el expediente respectivo, acreditando la causa de utilidad pública con base en dictámenes técnicos.

b) La declaratoria de utilidad debe publicarse en el Diario Oficial de la Federación (DOF) y en un diario de la localidad de que se trate, notificándose además personalmente a los titulares de los bienes a afectar. Únicamente en caso de que no se tengan identificados a los dueños o se ignore su domicilio o su localización, una segunda publicación en el $D O F$, dentro de los cinco días posteriores a la primera, surtirá efectos de notificación

c) Los interesados cuentan con un plazo de 15 días hábiles a partir de la notificación para manifestar ante la Secretaría correspondiente lo que a su derecho convenga y presentar pruebas.

d) Asimismo, la ley prevé la celebración de una audiencia para desahogo de pruebas, la que deberá verificarse en los ocho días hábiles posteriores a la presentación de las manifestaciones y, concluida la audiencia, las partes tendrán tres días para la presentación de sus alegatos.

e) Agotado el trámite de la citación para alegatos, la autoridad contará con un plazo de diez días hábiles para confirmar, modificar o revocar la declaratoria de utilidad pública.

f) Una vez confirmada o modificada la declaratoria de utilidad pública, el Ejecutivo federal debe decretar la expropiación en un plazo máximo de 30 días hábiles, contados a partir de que se haya dictado dicha resolución y si no lo hiciere, la declaratoria quedará sin efectos.

g) En contra de la determinación que confirme, modifique o revoque la causa de utilidad pública no existirá recurso administrativo y sólo procederá el juicio de amparo indirecto, cuya interposición interrumpirá el plazo de 30 días a que hace referencia el inciso anterior, hasta que se resuelva el mismo.

emitido el decreto respectivo, la declaratoria de utilidad pública quedará sin efectos. En caso de que se interponga el juicio de amparo, se interrumpirá el plazo a que se refiere esta fracción, hasta en tanto se dicte resolución en el mismo.

Artículo 3o. La Secretaría de Estado competente integrará y tramitará el expediente respectivo.

Cuando la promovente sea una entidad paraestatal, solicitará a la dependencia coordinadora de sector la emisión de la declaratoria. 
Por cuanto se refiere a la declaratoria específica de expropiación: ${ }^{17}$

a) Debe realizarse mediante decreto del Ejecutivo federal que se publicará en el $D O F$.

b) Los propietarios e interesados legítimos de los bienes y derechos afectados deberán ser notificados personalmente del decreto y del avalúo en que se fije el monto de la indemnización, dentro de los 15 días hábiles siguientes a la fecha de publicación del decreto.

c) Los afectados cuentan con un procedimiento judicial en el cual podrán controvertir únicamente el monto de la indemnización.

d) Una vez decretada la expropiación, ocupación temporal o limitación del dominio, se procederá a la ocupación inmediata del bien o impondrá la ejecución inmediata de dicha limitación.

e) La indemnización deberá pagarse en moneda nacional, o en especie si así se conviene, dentro de los 45 días hábiles siguientes a la publicación del decreto de expropiación.

f) La autoridad podrá proceder a la ocupación una vez que haya cubierto el monto fijado en el avalúo.

g) Toda vez que en caso de controversia, sólo estará en juego el monto de la indemnización, la ley prevé que la interposición de cualquier medio de defensa no suspenderá la ocupación del bien o la limitación del dominio.

Por otra parte, la ley prevé que no procede la audiencia previa en los casos siguientes: ${ }^{18}$

1. La construcción de obras de infraestructura pública y la prestación de servicios públicos que requieran de bienes inmuebles y sus mejoras,

17 Artículo 4o. Procederá la expropiación previa declaración de utilidad pública a que se refiere el artículo anterior.

18 Artículo 8o. En los casos a que se refieren las fracciones III bis, V, VI y X del artículo 1o. de esta Ley, el Ejecutivo federal hará la declaratoria de utilidad pública, decretará la expropiación, ocupación temporal o limitación de dominio y ordenará la ejecución inmediata de la medida de que se trate. Tratándose de la expropiación, no será aplicable lo dispuesto en las fracciones III a VII del artículo 2o. de esta Ley.

Esta resolución no admitirá recurso administrativo alguno y solamente podrá ser impugnada a través del juicio de amparo.

En los casos a que se refiere el primer párrafo de este artículo, durante la tramitación del juicio de amparo que en su caso se instaure, no podrá suspenderse la ejecución de la expropiación, la ocupación temporal o la limitación de dominio. 
derivada de concesión, contrato o cualquier acto jurídico celebrado en términos de las disposiciones legales aplicables.

2. La satisfacción de necesidades colectivas en caso de guerra o trastornos interiores; el abastecimiento de las ciudades o centros de población, de víveres o de otros artículos de consumo necesario, y los procedimientos empleados para combatir o impedir la propagación de epidemias, epizootias, incendios, plagas, inundaciones u otras calamidades públicas.

3. Los medios empleados para la defensa nacional o para el mantenimiento de la paz pública.

4. Las medidas necesarias para evitar la destrucción de los elementos naturales y los daños que la propiedad pueda sufrir en perjuicio de la colectividad.

En tales casos, la Ley prevé que la resolución sólo podrá ser impugnada a través del juicio de amparo; además, durante la tramitación del mismo no podrá suspenderse la ejecución de la expropiación, la ocupación temporal o la limitación del dominio.

\section{OPINIÓN}

Frente al dilema de absolutos que supone la postura anterior del más alto tribunal — de negación de la audiencia previa — y la actual — de escuchar en todos los casos previamente al afectado-, la reforma opta por una solución intermedia, en donde se otorga audiencia previa en la mayoría de los casos y la deniega en casos de suma urgencia, cuya ejecutividad resulta poco discutible y que pensamos puede resistir la aplicación de un test de razonabilidad.

Además, la reforma en el caso de la audiencia previa opta por un procedimiento administrativo breve, pero suficiente para cumplir con la garantía del debido proceso legal, en donde se notifica a los afectados el inicio del procedimiento y se les permite argumentar, presentar pruebas, desahogarlas en una audiencia y formular alegatos, contemplando ade- 
más el dictado de una resolución final debe confirmar, modificar o revocar la declaración de causa de utilidad pública. ${ }^{19}$

También resulta interesante la división del procedimiento en dos etapas claramente diferenciadas. En la primera el objeto es la demostración de la utilidad pública y los conflictos, en caso de que existan, versarán exclusivamente en la actualización de la hipótesis legal de utilidad pública en la que la declaración se funda.

Contra la determinación que confirme, modifique o revoque la causa de utilidad pública no existirá recurso administrativo y sólo procederá el juicio de amparo indirecto.

Esta es una situación muy interesante, puesto que la Ley opta por no acudir a la instancia contenciosa administrativa, tal vez sabedora de la celeridad que caracteriza a este tipo de juicios en nivel federal y que pueden tardar años en resolverse. Se opta por abrir la vía del amparo indirecto de manera inmediata, que es precisamente el sistema que operaba, antes de la creación del juicio contencioso administrativo por vía jurisprudencial en la Segunda Sala, lo cual desde luego no constituye una situación atípica. De hecho es interesante que estando el ejercicio de la facultad expropiatoria en juego se escoja un camino más rápido que, en principio, no está estructurado de la misma manera para otro tipo de actos administrativos, y que se ha hecho optativo para los particulares a través de una interpretación jurisprudencial.

En la segunda etapa del procedimiento, una vez demostrada la utilidad pública, se realizará el decreto expropiatorio y la contienda, en que caso de que se actualice, versará sobre el monto de la indemnización, sin que, por lo tanto, los medios de defensa puedan provocar la suspensión de la ocupación, en tanto que al haber quedado demostrada la causa de utilidad pública el procedimiento versará exclusivamente sobre el monto de la indemnización, por lo que no se justifica la paralización de la ocupación.

Por último, una innovación interesante del procedimiento consiste en la regulación de un nuevo supuesto cuando exista un cuestionamiento de la titularidad del bien o del derecho expropiado, pues en estos casos la indemnización será depositada y puesta a disposición de la autoridad que

19 Con estos supuestos se cumple con los requisitos señalados en la jurisprudencia P./J. 47/95, de rubro: "FORMALIDADES ESENCIALES DEL PROCEDIMIENTO. SON LAS QUE GARANTIZAN UNA ADECUADA Y OPORTUNA DEFENSA PREVIA AL ACTO PRIVATIVO”. 
conozca del recurso respectivo, para que ésta en su momento las asigne a sus legítimos propietarios, en el monto correspondiente.

Consideramos que se trata de una buena reforma que logra un importante equilibrio entre los derechos sociales y los derechos individuales que se colocan en conflicto por la expropiación. También se destaca la utilización de la libertad de configuración del legislador, quien no acepta como dogma la jurisprudencia de la Suprema Corte e innova una nueva solución con elementos fuertes que estamos seguros podrán resistir un juicio de constitucionalidad. 